Research Article

\title{
Sensitivity Study on Optimizing Thickness Distribution for a Membrane Reflector
}

\author{
He Huang $\mathbb{D}^{1}$ and Fu-ling Guan ${ }^{2}$ \\ ${ }^{1}$ School of Mechanics, Civil Engineering and Architecture, Northwestern Polytechnical University, Xi'an 710072, China \\ ${ }^{2}$ College of Civil Engineering and Architecture, Zhejiang University, Hangzhou 310058, China
}

Correspondence should be addressed to He Huang; hehuang0623@nwpu.edu.cn

Received 14 July 2019; Revised 22 September 2019; Accepted 17 October 2019; Published 23 November 2019

Academic Editor: Linda L. Vahala

Copyright ( 2019 He Huang and Fu-ling Guan. This is an open access article distributed under the Creative Commons Attribution License, which permits unrestricted use, distribution, and reproduction in any medium, provided the original work is properly cited.

\begin{abstract}
Inflatable membrane reflectors are widely used in space and terrestrial deployable antennas. The mechanical properties of the inflated membrane reflector, however, are often a limiting factor in the level of the surface accuracy that can be achieved. In this study, membrane structural analysis is combined with sizing optimization to tailor the thickness of the membrane to improve surface accuracy of an axisymmetric parabolic inflated membrane reflector. Two reflector surface accuracy evaluation methods are employed in the optimizer and researched about their effect on the sensitivity analysis. Gradient-based optimization is employed together with a simplified sensitivity analysis, and the resulting algorithm is demonstrated on a membrane reflector case study. A linear thickness filter is used to avoid checkerboard patterns, and optimized solutions are computationally shown to achieve a feasible level of surface accuracy.
\end{abstract}

\section{Introduction}

Inflatable reflector concepts have been considered and employed for the space and terrestrial deployable antennas, including QUASAT [1], IN-STEP [2], ARISE [3], NEXRAD [4], and other missions [5], due to their ultra-lightweight and high package ratio. These characteristics allow the scale of antennas to expand substantially while reducing the cost for launch, as compared with other types of deployable antennas. However, the mechanical properties of inflatable membrane structures restrict the range of the microwave bandwidth that can be achieved and, therefore, the range of application of these antennas [5].

The surface accuracy of the reflector is one of the most important factors influencing the gain and microwave bandwidth of the antenna, attracting the attention of previous research [6]. The so-called W-profile error, defined as the deviation from a parabolic profile due to the inflation, leads to a reduction in surface accuracy of the inflated membrane reflector. Jerkins and Marker [7] have studied the reflector figure error from plane sheet to curved surface, while
Greschik et al. [8-11] have studied the structural properties of the flat and curved axisymmetric membranes for the inflated reflectors and the initially approximate and exact parabolic shape design with different parametric assembling. In particular, in Ref. [10], the sensitivity study of the membrane reflectors has been conducted and stated that nonuniform thickness perturbations have effects on reflector surface errors. Naboulsi [12] has investigated geometric imperfections associated with inflated structures using geometric nonlinear finite element (FE). Kitano and Ishida [13] have obtained the discrete distribution of optimum thickness for circular membrane reflector. In order to decrease the W-profile error, DeSmidt et al. and Hill et al. $[14,15]$ have developed active control of the reflector using optimized gore/seam cable-actuated shape control. Coleman et al. [16] have studied the effects of the elastic tendon boundary support and reflector dimensions on surface accuracy of the inflatable antenna by an ideal parabolic form and two different flat panel design patterns. Bouzidi and Lecieux [17] have optimized the shape parameter design of initial geometry for a space inflatable membrane reflector by a numerical 
downhill simplex method. Wang et al. [18] have employed a kind of support ribs stretched by the tension system to improve the surface accuracy of the inflatable deployable reflector. Huang and Guan [5] have proposed an iterative method working from the initial profile solution for reflector precision improvement.

There is no gainsaying that these methods have improved surface accuracy, but error magnitudes are still relatively large. Meanwhile, 3D printing technology is being processed in the manufacturing industry, but this application is not used in membrane reflector and relative sensitivity study is seldom carried on deeply. This has motivated the current study focusing on membrane reflector sensitivity and using optimization to tailor the membrane thickness to further enhance surface accuracy. There are many optimization methods for wrinkle-free design of membrane structures such as stress-based topology optimization [19], multimaterial topology optimization approach [20], and global shape optimization [21]. Although wrinkles are avoided in membrane reflector by engineering means such as changing border forms, the thoughts and methods of optimization and sensitivity analysis can be referred. In particular, we use the Method of Moving Asymptotes (MMA) by Svanberg [22], an optimizer that is widely used by the topology optimization community [23], together with the adjoint method for much more specific sensitivity analysis compared with Ref. [10]. The design variables are the thicknesses of membrane elements, and the objective function is the minimum surface accuracy of inflated reflector. The inflated reflector deformation is computed by using the finite element method.

The remainder of this paper is organized as follows. The importance and foundation of two surface accuracy evaluation methods for the objective function are discussed in Section 2. The sizing optimization formulation is stated in Section 3, and the sensitivity analysis based on two methods is presented in Section 4. A membrane reflector case study is used to illustrate the method in Section 5, with concluding remarks following in Section 6.

\section{Surface Accuracy}

One of the most important parameters for the antenna is the antenna gain, defined as the ratio of the power transmitted by the antenna to the power of an ideal isotropic radiator. With the mean phase plane chosen as the phase reference plane and the simplification during the prediction of the gain reduction and pattern degradation, the reflector gain of the Ruze equation [24] is given as

$$
G=G_{0} e^{-\delta^{2}}=\eta\left(\frac{\pi D}{\lambda}\right)^{2} e^{-(4 \pi \sigma / \lambda)^{2}}
$$

where $G_{0}$ is the no-error gain axial value as $\eta(\pi D / \lambda)^{2}, \delta$ is a statistical phase error calculated from the mean phase plane, $\eta$ is the aperture efficiency, $D$ is the diameter of the reflector aperture, $\lambda$ is the wavelength, and $\sigma$ is the effective reflector tolerance as the structural parameter to approximately quantify the reflector surface efficiency.
When the effective gain is described in units of decibels $(\mathrm{dB})$, the equation can be expanded as [6]

$$
G=10 \log _{10} \eta\left(\frac{\pi D}{\lambda}\right)^{2}-4.3429\left(\frac{4 \pi \sigma}{\lambda}\right)^{2}
$$

From this expression, it is clear that the effective gain can be kept large if $\sigma$ is kept as low as possible. Therefore, the evaluation of this effective reflector tolerance $\sigma$ is important and plays a significant role in the objective function and sensitivity analysis of reflector optimization.

2.1. HPL-E Method. Generally, the interpretation of the parameter $\sigma$ is presented as the Root Mean Square HalfPath-Length Error (HPL-E) Method of the microwave rays distributed over and reflected by the surface [6]. This is considered to be the most significant of all these separate efficiency terms and consequently motivates the use of root mean square (RMS), familiarly surface accuracy, as the objective function for the optimization formulation.

Based on the surface tolerance mentioned in Ref. [6], the HPL-E $\varepsilon$ of an arbitrary point $i(i=1,2, \cdots, n)$ on the reflector can be derived by the geometric path length analysis as

$$
\varepsilon_{i}=r_{i z} \mathbf{n}_{i} \mathbf{u}_{i},
$$

where $\mathbf{n}_{i}$ is the normal vector on the point $i$ as $\left[r_{i x}, r_{i y}, r_{i z}\right]$ (assume axis $Z$ is the focus axis), $\mathbf{d}_{i}$ is the displacement vector of the point $i$ as $\mathbf{u}_{i}=\left[u_{i}, v_{i}, w_{i}\right]^{\mathrm{T}}$, and $r_{i z}$ is the value of normal vector in the axial direction. It means that the HPL-E is the product of the normal value of displacement vector and the value of normal vector in the axial direction.

There is a best-fit parabolic profile, namely, the mean phase plane, of each deformed surface as the phase reference plane during the tolerance theory deriving [6]. Depending on this phase reference plane, a new half-path-length error $\varepsilon^{*}$ can be computed as

$$
\varepsilon_{i}^{*}=r_{i z} \mathbf{n}_{i}\left(\mathbf{u}_{i}+\Delta \mathbf{u}_{i}\right)=\left[\begin{array}{lll}
a_{i} & b_{i} & c_{i}
\end{array}\right]\left(\left[\begin{array}{c}
u_{i} \\
v_{i} \\
w_{i}
\end{array}\right]+\left[\begin{array}{c}
\Delta u_{i} \\
\Delta v_{i} \\
\Delta w_{i}
\end{array}\right]\right),
$$

where $a_{i}, b_{i}$, and $c_{i}$ are $r_{i z} r_{i x}, r_{i z} r_{i y}$, and $r_{i z}^{2}$, respectively, as aforementioned; the vector $\Delta \mathbf{u}_{i}=\left[\Delta u_{i}, \Delta v_{i}, \Delta w_{i}\right]^{\mathrm{T}}=$ $\left[-u_{0}-z \theta_{y},-v_{0}-z \theta_{x},-w_{0}-k z-y \theta_{x}+x \theta_{y}\right]^{\mathrm{T}}$ is the displacement difference between the best-fit parabolic profile and the original parabolic profile. Thereinto, the best-fit parabolic profile usually can be determined by six parameters, i.e., translations $u_{0}, v_{0}$, and $w_{0}$ in three axes, rotations $\theta_{x}$ and $\theta_{y}$ along the axis $X$ and $Y$, respectively, and focus-length change parameter $k=F / F_{0}-1$. 
Thus, there is a Root Mean Square HPL-E $\sigma$ depending on the best-fit parabolic profile as

$$
\sigma=\sqrt{\frac{\sum_{i=1}^{n}\left(\varepsilon_{i}^{*}\right)^{2}}{n}}
$$

where $n$ is the total number of the points on the reflector. This $\sigma$, the so-called RMS, is an important indicator for structural engineers to evaluate the reflector efficiency as aforementioned in this section.

2.2. BFP-E Method. The HPL-E Method is a kind of traditional method to evaluate the reflector efficiency. It is apparently dependent on the displacement vector $\mathbf{u}$ which is supposed to be tiny as shown in equation (4). When it comes to high-precision reflector, this HPL-E Method should be reconsidered to discuss its effectivity for the reflector surface accuracy evaluation.

The membrane reflector loaded by the inflation pressure has large displacements that cause the geometry nonlinear property. In other words, it might be improper to use the HPL-E Method with this large displacement vector to indicate the reflector efficiency of membrane structures. One more problem is the parameter, specifically the thickness here in the following sensitivity analysis, influencing the structural property and displacement, which means that it might be not comparable for each optimal result by using the HPL-E Method.

Therefore, another method, namely, the Best-Fit-Profile Error (BFP-E) Method, is stated here to evaluate the reflector surface accuracy. The fundamental theory of the BFP-E Method is to find a best-fit parabolic profile for a deformed reflector and get the RMS of the deviation error between the deformed reflector and the best-fit profile. It could indicate the surface roughness for the deformed surface without the displacement vector.

The specific steps of the BFP-E Method are explained as follows. Firstly, it is easy to get the best-fit profile computed from the deformed reflector by several algorithms, like the least square method. Secondly, a best-fit-profile error $\varepsilon^{\#}$ can be defined as the norm of deviation vector for each point and shown as

$$
\varepsilon_{i}^{\#}=\left|\Delta \mathbf{x}_{i}\right|=\left|\mathbf{x}_{i}^{\mathrm{DP}}-\mathbf{x}_{i}^{\mathrm{BFP}}\right|,
$$

where $\mathbf{x}_{i}{ }^{\text {DP }}$ and $\mathbf{x}_{i}{ }^{\text {BFP }}$ are the coordinates of the deformed profile and the best-fit profile. Finally, a Root Mean Square BFP-E $\sigma$ can be calculated by substituting $\varepsilon^{\#}$ into equation (5).

It is obvious that this method is not exactly precise as the HPL-E Method since the BFP-E Method is based on the concept of geometry surface instead of phase plane. However, this method can indicate the deformation state for any reflectors with the uniform quantification standard. It is convenient to conduct the following sensitivity research by utilizing this evaluation of the reflector efficiency.

\section{Optimization Model}

The optimization formulation uses surface accuracy, namely, the RMS $\sigma$ based on two methods (HPL-E Method and BFPE Method), as the objective function. The thickness $t_{e}$ of each membrane element is the (dependent) design variables, which are expressed as a function of the independent design variables $\varphi$ as described below. The considered reflector is assumed to be an axisymmetric parabolic reflector made of isotropic membrane material and loaded by inflation pressure. The final deformed shape of the reflector under this pressure is influenced by the stiffness and therefore the thickness of the membrane. The optimization problem can be written as

$$
\begin{aligned}
& \text { find } \boldsymbol{\varphi}=\left[\begin{array}{lllll}
\varphi_{1} & \cdots & \varphi_{j} & \cdots & \varphi_{m}
\end{array}\right] \text {. } \\
& \underset{\mathbf{t}_{e}(\boldsymbol{\varphi})}{\operatorname{minimize}} \sigma\left(\boldsymbol{t}_{e}(\boldsymbol{\varphi})\right)=\sqrt{\frac{\sum_{c}^{n}\left(\varepsilon_{i}\right)^{2}}{n}} \\
& \text { subject to } \quad \boldsymbol{K}\left(\boldsymbol{t}_{e}(\boldsymbol{\varphi}), \boldsymbol{u}\right) \boldsymbol{u}-\boldsymbol{F}(\boldsymbol{u})=\mathbf{0} \\
& t_{\min } \leq t_{e}(\boldsymbol{\varphi}) \leq t_{\max },
\end{aligned}
$$

where $m$ is the number of independent design variables, $t_{\text {min }}$ and $t_{\max }$ are the lower and upper bounds on the thickness of a membrane element, respectively, $\mathbf{K}$ is the global stiffness matrix for the membrane structure, $\mathbf{u}$ is the nodal displacement vector, and $\mathbf{F}$ is the nodal load vector. Note that the stiffness and applied pressure loads are functions of the displacement field $\mathbf{u}$, and thus, we have a geometrically nonlinear problem that is solved iteratively herein using the Newton-Raphson Method [25].

Here in equation (7), $\varepsilon$ will be separately discussed as HPL-E $\varepsilon^{*}$ and BFP-E $\varepsilon^{\#}$ by the HPL-E Method and the BFP-E Method, respectively, to conduct two optimization models. The results of the best RMS $\sigma_{\text {HPL-E }}$ and $\sigma_{\mathrm{BFP}-\mathrm{E}}$ can be computed for each optimization model to evaluate the optimal surface accuracy.

It is well known in the structural topology optimization community that using low-order finite elements, such as linear three node triangles, may lead to artificial solutions known as checkerboards. The checkerboard pattern consists of alternating solid and void elements or equivalently alternating elements with high and low thickness. The stiffness of this pattern is overestimated with low-order finite elements [26]. Density filters in topology optimization [25, 27, 28] are a popular means for circumventing this effect, in addition to eliminating a related issue of solution mesh dependency. We employ this idea here to relate the independent design variables $\varphi$ to the thickness of membrane elements $\mathbf{t}^{e}$ and refer to it as thickness filtering, although the logic is exactly the same as the density filter.

Defining $r_{\text {min }}$ as the radius of the thickness filter (over which the smoothing occurs), we denote the neighborhood set of an element $e$ as $N^{e}$ and define it as [28]

$$
j \in N^{e}, \quad \text { if }\left\|\mathbf{x}_{j}-\mathbf{x}^{e}\right\| \leq r_{\min }
$$


where $\mathbf{x}_{j}$ is the location of design variable $j$ and $\mathbf{x}^{e}$ is the location of the centroid of $e$.

These new design variables $\varphi_{j}$ are then filtered onto the elemental thickness as

$$
t_{e}=\frac{\sum_{j \in N^{e}} \varphi_{j} \omega\left(\mathbf{x}_{j}-\mathbf{x}^{e}\right)}{\sum_{j \in N^{e}} \omega\left(\mathbf{x}_{j}-\mathbf{x}^{e}\right)},
$$

where $\omega$ is the linear distance-based weighting function

$$
\omega\left(\mathbf{x}_{j}-\mathbf{x}^{e}\right)= \begin{cases}\frac{r_{\min }-\left\|\mathbf{x}_{j}-\mathbf{x}^{e}\right\|}{r_{\min }}, & \text { if } j \in N^{e}, \\ 0, & \text { otherwise. }\end{cases}
$$

The Method of Moving Asymptotes [22] is used to solve this problem with sensitivities computed as described in the following section.

\section{Sensitivity Analysis}

The adjoint method is used to estimate the sensitivities of objective function [23]. The equilibrium condition is added to the objective function as

$$
c_{A}=\sigma(\mathbf{u})-\lambda^{\mathrm{T}}\left[\mathbf{K}\left(t_{e}(\varphi), \mathbf{u}\right) \mathbf{u}-\mathbf{F}(\mathbf{u})\right],
$$

where $\lambda^{\mathrm{T}}$ is any arbitrary vector.

The derivatives of the adjoint function in equation (11) is given as

$$
\begin{aligned}
\frac{\partial c_{A}}{\partial t_{e}}= & \frac{\partial \sigma}{\partial\left(\mathbf{u}^{\mathrm{T}}\right)} \frac{\mathrm{d} \mathbf{u}}{\mathrm{d} t_{e}}-\lambda^{\mathrm{T}}\left[\left(\frac{\partial \mathbf{K}}{\partial\left(\mathbf{u}^{\mathrm{T}}\right)} \frac{\mathrm{d} \mathbf{u}}{\mathrm{d} t_{e}}+\frac{\partial \mathbf{K}}{\partial t_{e}}\right) \mathbf{u}\right. \\
& \left.+\mathbf{K} \frac{\mathrm{d} \mathbf{u}}{\mathrm{d} t_{e}}-\frac{\partial \mathbf{F}}{\partial\left(\mathbf{u}^{\mathrm{T}}\right)} \frac{\mathrm{d} \mathbf{u}}{\mathrm{d} t_{e}}\right] .
\end{aligned}
$$

Grouping like terms gives

$\frac{\partial c_{A}}{\partial t_{e}}=-\lambda^{\mathrm{T}} \frac{\partial \mathbf{K}}{\partial t_{e}} \mathbf{u}+\left\{\frac{\partial \sigma}{\partial\left(\mathbf{u}^{\mathrm{T}}\right)}-\lambda^{\mathrm{T}}\left[\frac{\partial \mathbf{K}}{\partial\left(\mathbf{u}^{\mathrm{T}}\right)} \mathbf{u}+\mathbf{K}-\frac{\partial \mathbf{F}}{\partial\left(\mathbf{u}^{\mathrm{T}}\right)}\right]\right\} \frac{\mathrm{d} \mathbf{u}}{\mathrm{d} t_{e}}$,

where we note

$$
\begin{aligned}
\frac{\partial \mathbf{K}}{\partial\left(\mathbf{u}^{\mathrm{T}}\right)} \mathbf{u}+\mathbf{K}-\frac{\partial \mathbf{F}}{\partial\left(\mathbf{u}^{\mathrm{T}}\right)} & =\frac{\partial \mathbf{K u}+\mathbf{K} \partial \mathbf{u}-\partial \mathbf{F}}{\partial\left(\mathbf{u}^{\mathrm{T}}\right)} \\
& =\frac{\partial(\mathbf{K u}-\mathbf{F})}{\partial\left(\mathbf{u}^{\mathrm{T}}\right)}=\mathbf{K}_{G}
\end{aligned}
$$

and define $\mathbf{K}_{G}$ the geometric stiffness matrix at the last iteration of the structural analysis [25].

In order to eliminate the last term in equation (13), the arbitrary adjoint variables $\lambda^{\mathrm{T}}$ are chosen to solve

$$
\frac{\partial \sigma}{\partial\left(\mathbf{u}^{\mathrm{T}}\right)}=\lambda^{\mathrm{T}} \mathbf{K}_{G}
$$

The derivative then simplifies to

$$
\frac{\partial c_{A}}{\partial t_{e}}=-\lambda^{\mathrm{T}} \frac{\partial \mathbf{K}}{\partial t_{e}} \mathbf{u},
$$

where the derivative of the stiffness matrix with respect to the membrane thickness is given as

$$
\frac{\partial \mathbf{K}\left(t_{e}\right)}{\partial t_{e}}=\mathbf{K}_{e 0},
$$

where $\mathbf{K}_{e 0}$ is the element stiffness matrix for element $e$ having unit thickness. This provides all of the terms needed to compute the derivatives in equation (16).

The derivatives of equation (16) with respect to the independent optimization variables, which are needed by the optimizer, are then simply given by the chain rule [27] as

$$
\frac{\partial c_{A}}{\partial \varphi_{i}}=\sum_{e \in \Omega} \frac{\partial c_{A}}{\partial t_{e}} \frac{\partial t_{e}}{\partial \varphi_{i}}
$$

where

$$
\frac{\partial t_{e}}{\partial \varphi_{i}}=\frac{w\left(\mathbf{x}_{i}-\mathbf{x}^{e}\right)}{\sum_{j \in N^{e}} w\left(\mathbf{x}_{j}-\mathbf{x}^{e}\right)} .
$$

When computing adjoint variables $\lambda^{\mathrm{T}}$ in equation (16), the left term of equation (15) should be considered. Hence, the RMS $\sigma$ based on the HPL-E Method and the BFP-E Method has different expression shown as the following.

4.1. HPL-E Derivative. The partial derivatives of RMS HPL-E $\sigma$ with respect to the displacements $\mathbf{u}$ are needed to solve $\lambda^{\mathrm{T}}$, as indicated in equation (15). This can be computed as

$$
\frac{\partial \sigma_{\mathrm{HPL}-\mathrm{E}}}{\partial\left(\mathbf{u}^{\mathrm{T}}\right)}=\left[\frac{\sum_{i=1}^{n}\left(\varepsilon_{i}{ }^{*}\right)^{2}}{n}\right]^{-1 / 2} \cdot \frac{1}{n} \cdot \sum_{i=1}^{n} \frac{\partial\left(\varepsilon_{i}{ }^{*}\right)}{\partial\left(\mathbf{u}^{\mathrm{T}}\right)},
$$

where the displacement vector $\mathbf{u}$ is listed in sequence as

$$
\begin{aligned}
& \mathbf{u}=\left[\begin{array}{lllllllllll}
u_{1} & v_{1} & w_{1} & \cdots & u_{i} & v_{i} & w_{i} & \cdots & u_{n} & v_{n} & w_{n}
\end{array}\right] \\
& =\left[\begin{array}{lllllllllll}
d_{1} & d_{2} & d_{3} & \cdots & d_{3 i-2} & d_{3 i-1} & d_{3 i} & \cdots & d_{3 n-2} & d_{3 n-1} & d_{3 n}
\end{array}\right] \text {, }
\end{aligned}
$$


where $d$ is the displacements listed by the order of the degree of freedom (DOF) and has the mapping relationship with $\mathbf{u}_{i}=\left[u_{i}, v_{i}, w_{i}\right]^{\mathrm{T}}$.

Here in equation (4), it is also noted that the six parameters for each best-fit parabolic profile are unique so that the vector $\Delta \mathbf{u}_{i}=\left[\Delta u_{i}, \Delta v_{i}, \Delta w_{i}\right]^{\mathrm{T}}$ can be determined by the parameters and have no relationship with the displacement vector u. So equation (20) can be rewritten more simply according to the DOF for each element as

$$
\begin{aligned}
\frac{\partial \sigma_{\mathrm{HPL}-\mathrm{E}}}{\partial d_{k}} & =\frac{\sigma_{\mathrm{HPL}-\mathrm{E}}^{-1}}{n} \sum_{i=1}^{n} \frac{\partial\left(a_{i} u_{i}+b_{i} v_{i}+c_{i} w_{i}\right)}{\partial d_{k}} \\
& =\frac{\sigma_{\mathrm{HPL}-\mathrm{E}}^{-1}}{n} \sum_{i=1}^{n} \frac{\partial\left(a_{i} d_{3 i-2}+b_{i} d_{3 i-1}+c_{i} d_{3 i}\right)}{\partial d_{k}} .
\end{aligned}
$$

This simplification shows that the displacement has no relationship with the original profile and the best-fit parabolic profile. It is linear in this derivative.

4.2. BFP-E Derivative. The partial derivatives of RMS BFP-E $\sigma$ with respect to the displacements $\mathbf{u}$ can be computed as

$$
\begin{aligned}
\frac{\partial \sigma_{\mathrm{BFP}-\mathrm{E}}}{\partial\left(\mathbf{u}^{\mathrm{T}}\right)} & =\left[\frac{\sum_{i=1}^{n}\left(\varepsilon_{i}^{\#}\right)^{2}}{n}\right]^{-1 / 2} \cdot \frac{1}{n} \cdot \sum_{i=1}^{n} \frac{\partial\left(\varepsilon_{i}^{\#}\right)}{\partial\left(\mathbf{u}^{\mathrm{T}}\right)} \\
& =\frac{\sigma_{\mathrm{BFP}-\mathrm{E}}{ }^{-1}}{n} \cdot \sum_{i=1}^{n} \frac{\partial\left(\left|\mathbf{x}_{i}^{\mathrm{DP}}-\mathbf{x}_{i}^{\mathrm{BFP}}\right|\right)}{\partial\left(\mathbf{u}^{\mathrm{T}}\right)} .
\end{aligned}
$$

Considering equation (21), equation (23) can be rewritten more simply according to the DOF for each element as

$$
\begin{aligned}
\frac{\partial \sigma_{\mathrm{BFP}-\mathrm{E}}}{\partial d_{k}}= & \frac{\sigma_{\mathrm{BFP}-\mathrm{E}}{ }^{-1}}{n} \sum_{i=1}^{n}\left(x_{i}^{\mathrm{DP}}-x_{i}^{\mathrm{BFP}}\right) \frac{\partial x_{i}^{\mathrm{DP}}}{\partial d_{k}} \\
& +\left(y_{i}^{\mathrm{DP}}-y_{i}^{\mathrm{BFP}}\right) \frac{\partial y_{i}^{\mathrm{DP}}}{\partial d_{k}}+\left(z_{i}^{\mathrm{DP}}-z_{i}^{\mathrm{BFP}}\right) \frac{\partial z_{i}^{\mathrm{DP}}}{\partial d_{k}} .
\end{aligned}
$$

An assumption is introduced here as

$$
\begin{aligned}
& \frac{\partial x_{i}^{\mathrm{DP}}}{\partial d_{k}}= \begin{cases}1, & \text { if } k=3 i-2, \\
0, & \text { otherwise, }\end{cases} \\
& \frac{\partial y_{i}^{\mathrm{DP}}}{\partial d_{k}}= \begin{cases}1, & \text { if } k=3 i-1, \\
0, & \text { otherwise, }\end{cases} \\
& \frac{\partial z_{i}^{\mathrm{DP}}}{\partial d_{k}}= \begin{cases}1, & \text { if } k=3 i, \\
0, & \text { otherwise, }\end{cases}
\end{aligned}
$$

which means that the displacement has no relationship with the best-fit profile geometry and it is approximately linear in this derivative.

4.3. Optimization Process. The flowchart of this optimization is shown in Figure 1. Here, two optimization models are separately built by using the HPL-E Method and the BFP-E
Method to evaluate the inflated reflector surface accuracy as the objective function.

Hence, the HPL-E Model uses equations (4) and (20) for the optimization part while the BFP-E Model uses equations (6) and (23). But the results of each model can output the RMS $\sigma_{\mathrm{HPL}-\mathrm{E}}$ and $\sigma_{\mathrm{BPP}-\mathrm{E}}$ as the characterization of surface accuracy.

\section{Case Study}

5.1. Model Parameters. The proposed approaches are demonstrated on a reflector with target axisymmetric parabolic profile, which is utilized in its working state. The geometry parameters and the material properties of the target membrane reflector are listed in Table 1.

The finite element mesh is shown in Figure 2. The pressure load $P$ is $20 \mathrm{~Pa}$ applied on the concave side of the reflector, and the black points in this figure are assumed fixed nodes with all displacements set to zero.

The nodal coordinates for the initial profile are obtained from the Initial Profile FE Iterative Method, a trial-and-error approach, as described in Ref. [5]. The RMS BFP error of the deformed reflector based on this initial profile is $0.1489 \mathrm{~mm}$.

The optimization parameters are listed in Table 2. The radius $r_{\min }$ in this case is treated as the minimum radius of circle that can cover the biggest elements and it is additionally considered in Section 5.3 which is initially set to $65.789 \mathrm{~mm}$.

5.2. Result Comparison between Two Methods. As aforementioned, two different methods for reflector surface accuracy evaluation are employed in the reflector thickness optimization. Here, the optimal results by these two method models (using HPL-E $\varepsilon^{*}$ and BFP-E $\varepsilon^{\#}$ in equation (7)) are compared and discussed. The results of this case study optimized by the HPL-E Method and the BFP-E Method without considering the filtering are stated.

The results of best RMS $\sigma_{\mathrm{HPL}-\mathrm{E}}$ and $\sigma_{\mathrm{BFP}-\mathrm{E}}$ optimized by the HPL-E Model are $0.1067 \mathrm{~mm}$ and $0.4281 \mathrm{~mm}$, respectively. On the contrary, the results of best RMS $\sigma_{\mathrm{HPL}-\mathrm{E}}$ and $\sigma_{\text {BFP-E }}$ optimized by the BFP-E Model are $0.4508 \mathrm{~mm}$ and $0.0370 \mathrm{~mm}$, respectively. From the data, both two results improve its objective RMS $\sigma_{\mathrm{HPL}-\mathrm{E}}$ and $\sigma_{\mathrm{BFP}-\mathrm{E}}$ by the relevant method, respectively, and keep the other reference $\sigma_{\mathrm{BFP}-\mathrm{E}}$ and $\sigma_{\mathrm{HPL}-\mathrm{E}}$ stable. It is also indicated that the optimization based on the BFP-E Model is reasonably better than that based on the HPL-E Model.

Thus, the result of the BFP-E Model is more precise and suitable for reflector surface accuracy in this study. The section profile of inflated reflector based on this result is drawn in Figure 3. It is illustrated that the RMS $\sigma_{\mathrm{BFP}-\mathrm{E}}(0.0370 \mathrm{~mm})$ is significantly small so that the inflated reflector surface almost coincides with its best-fit profile parabolic surface. It is meant that the BFP-E Model solves the $\mathrm{W}$-profile error of inflated reflector in Ref. [5] by optimal thickness distribution in this study.

The optimal reflector based on the BFP-E Method is mainly focused on the thickness distribution of this membrane reflector. The Von Mises stress distribution and 


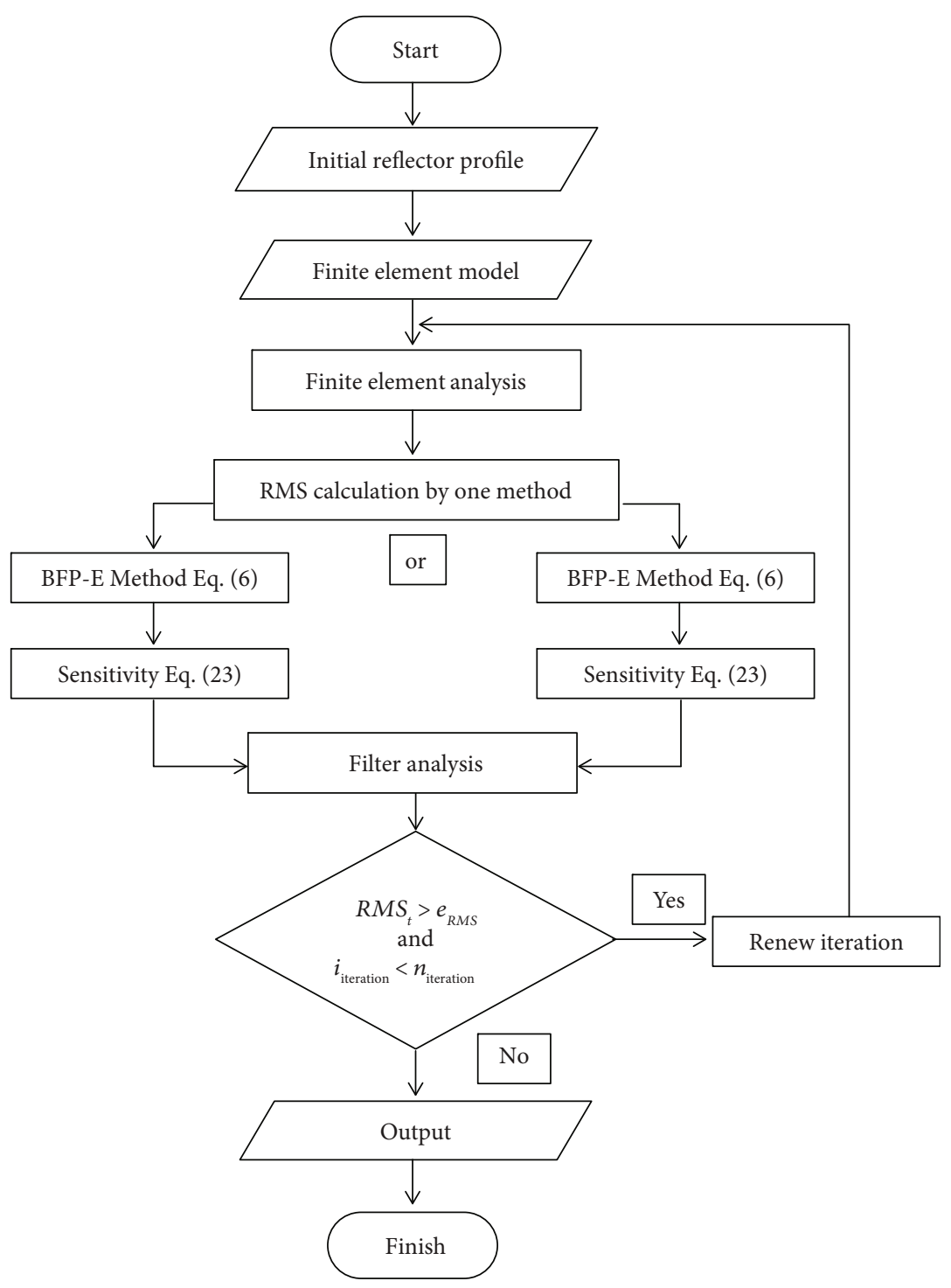

FIgURE 1: Optimization process.

TABLE 1: Geometry parameters and material properties.

\begin{tabular}{lc}
\hline Geometry parameters & $D=3200 \mathrm{~mm}$ \\
Diameter & $f=1500 \mathrm{~mm}$ \\
Focus length & $f / D=0.46875$ \\
Ratio of focus to diameter & \\
Material properties & $t_{0}=0.025 \mathrm{~mm}$ \\
Initial thickness & $E=2500 \mathrm{MPa}$ \\
Elastic modulus & $v=0.34$ \\
Poisson's ratio &
\end{tabular}

thickness distribution of the inflated reflector are drawn in Figures 4(a) and 4(b), respectively. It is shown that the stress decreases quickly then increases slightly from the center to circumference along the radial direction in Figure 4(a). The thickened elements at the middle ring in Figure 4(b) affect the stress distribution and the deformation of inflated reflec- tor so as to substantially get rid of the $\mathrm{W}$-profile error as shown in Figure 3 compared with Figures 3 and 4 in Ref. [5]. In addition, there are sudden changes among the outermost elements in Figure 4 since the outermost nodes are fixed as the boundary constraints so that there is stress concentration in these elements.

It is shown that the distribution range of Figure $4(\mathrm{~b})$ approaches the initial thickness $0.025 \mathrm{~mm}$ of the original model while the thickness only in the separated blue parts is concaved nearly to the initial thickness. The displacement vector $\mathbf{u}$ in equation (4) is taken into consideration during each iteration of the optimizing based on the BFP-E Method. On the one hand, the thick membrane can keep the displacement possibly little to get a small RMS $\sigma_{\text {HPL-E }}$. On the other hand, the optimizer decreases the thickness to search the potentially optimal solution. It is obviously contrary so that the final result becomes highly checkerboard but reasonable [27] for the optimization based on the MMA and without the filtering. 


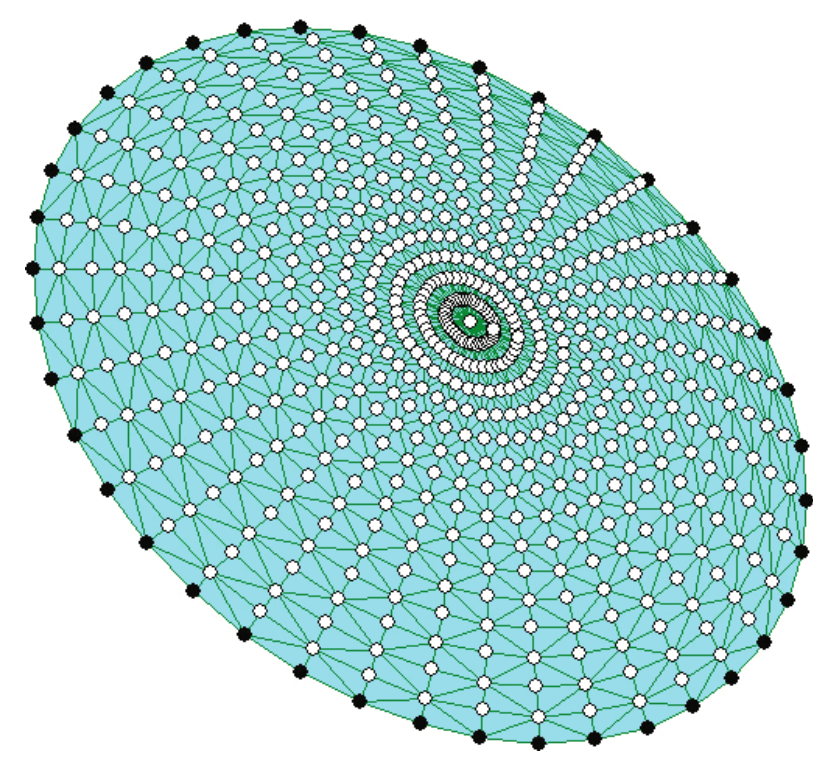

Figure 2: Finite element mesh.

TABLE 2: Optimization parameters.

\begin{tabular}{lc}
\hline Lower bound of thickness & $t_{0 \min }=0.020 \mathrm{~mm}$ \\
Upper bound of thickness & $t_{0 \max }=0.500 \mathrm{~mm}$ \\
Initial asymptote parameter & asyinit $=0.25$ \\
\hline
\end{tabular}

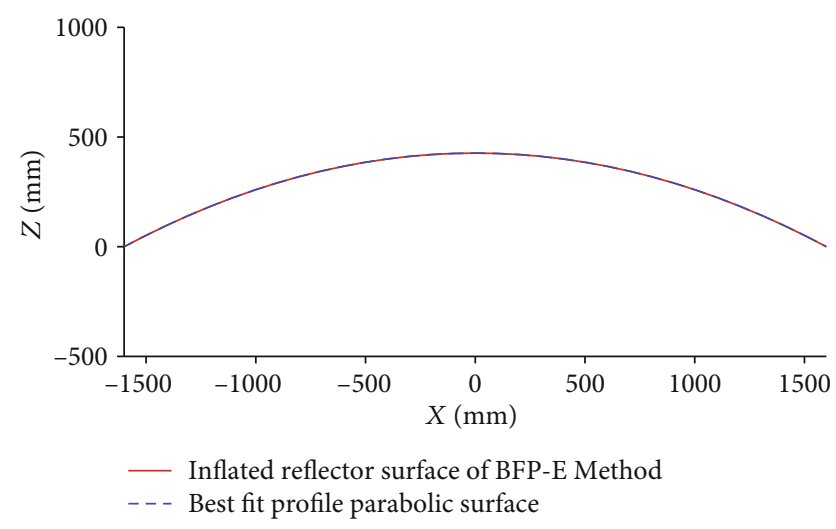

FIGURE 3: Section profile of inflated reflector of optimal result by the BFP-E Method.

Compared with the BFP-E Method, the Von Mises stress distribution and thickness distribution of optimal reflector by the HPL-E Method are shown in Figures 5(a) and 5(b), respectively. It is shown that the optimization converges on this highly checkerboard thickness distribution and the average thickness is much bigger than the BFP-E solution. The objective function $\sigma_{\text {HPL-E }}$ calculated by $\varepsilon^{*}$ in equation (4) has a direct relationship with the structural displacement $\mathbf{u}$, and the best-fit parabolic profile is fixed so that the displacement difference vector $\Delta \mathbf{u}_{i}$ assumption in equation (22) leads to the constant derivative. This kind of surface accuracy definition by the HPL-E Method precipitates the optimizing convergence on an abnormal solution. Hence, the HPL-E Method is not suitable for this kind of reflector surface accuracy evaluation.

5.3. Effect of Thickness Filter Radius. As shown in the results above, the thickness of reflector distribution matches quite well with the $\mathrm{W}$-error profile feature. But one of the disadvantages of this solution is that the thickness varies somewhat rapidly over the radius of the membrane (i.e., the gradient of thickness in the radial direction is relatively large).

The filter radius $r_{\min }$, however, offers a direct avenue to influence the gradient of the membrane thickness. On the basis of the BFP-E Method optimization, the corresponding RMS $\sigma_{\text {BFP-E }}$ and $\sigma_{\text {HPL-E }}$ magnitudes of optimal results are shown in Table 3 and the iteration curves of the optimization are drawn in Figure 6. It is illustrated that the surface accuracy $\sigma_{\mathrm{BFP}-\mathrm{E}}$ becomes worse with largening the filter radius and the oscillation of iteration tends to be smaller.

Figure 7 shows the optimized thickness distributions using several different magnitudes of the filter radius $r_{\min }$. The thickness distributions of Figure 7(a) clearly indicate that minimizing $R M S \sigma_{\mathrm{BFP}-\mathrm{E}}$ is achieved by varying thickness radially across the reflector. The thickness is smallest at the center of the reflector, then increases to form a stiff inner "ring," and then decreases until the boundary is reached, where thickness again increases to connect to the support structure.

From Figure 7 and Table 3, it is clearly seen that as the filter radius is increased, the membrane thickness distribution becomes smoother (smaller radial gradient in membrane thickness) and the optimized RMS $\sigma$ values increase. As the definition of $r_{\min }$ in Section 5.1, the bigger $r_{\min }$ is, the more elements are related to smooth the thickness because of radiant element distribution in this case. There is thus a direct tradeoff between the degree of thickness variation and the performance. Finally, we note that a filter radius of $r_{\min }=358.376 \mathrm{~mm}$ leads to a near uniform thickness distribution. From another perspective, the reasonable element size can improve the whole computation accuracy but has little effect on the optimal results of thickness distribution study.

\section{Conclusions}

This paper has used a gradient-based optimizer to substantially improve the surface accuracy of an axisymmetric parabolic inflated membrane reflector. Two methods, namely, Half-Path-Length Error Method and Best-Fit-Profile Error Method, are employed to evaluate the reflector surface accuracy and used in the derivatives of the optimizer. The thickness of the membrane elements was designated as the design variables using root mean square error based on these two evaluation methods as the objective function to be minimized. Sensitivities were computed via the adjoint method, with a geometrically nonlinear finite element analysis used to predict the deformed state under a pressure load. The thickness filtering was applied to research its effect.

Results demonstrated that the traditional HPL-E Method is not feasible here to evaluate the reflector surface accuracy. 


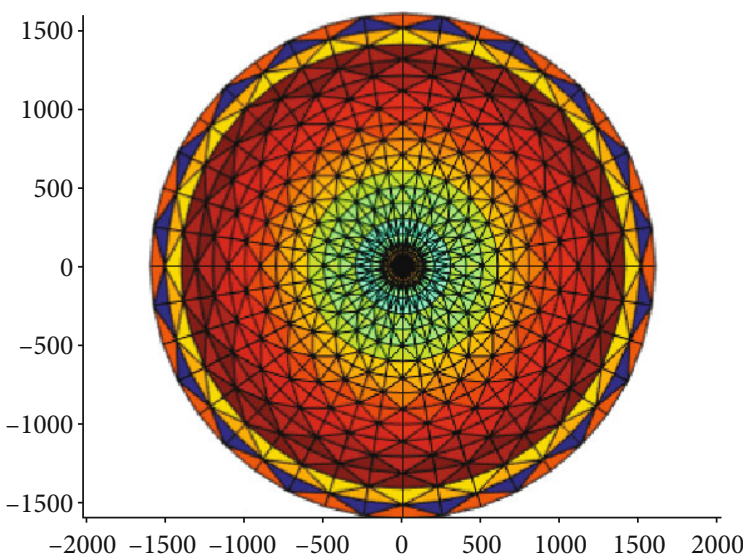

(a) Von Mises stress distribution

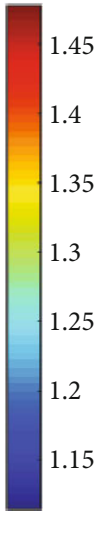

FiguRE 4: Stress and thickness distribution of optimal reflector by the BFP-E Method.

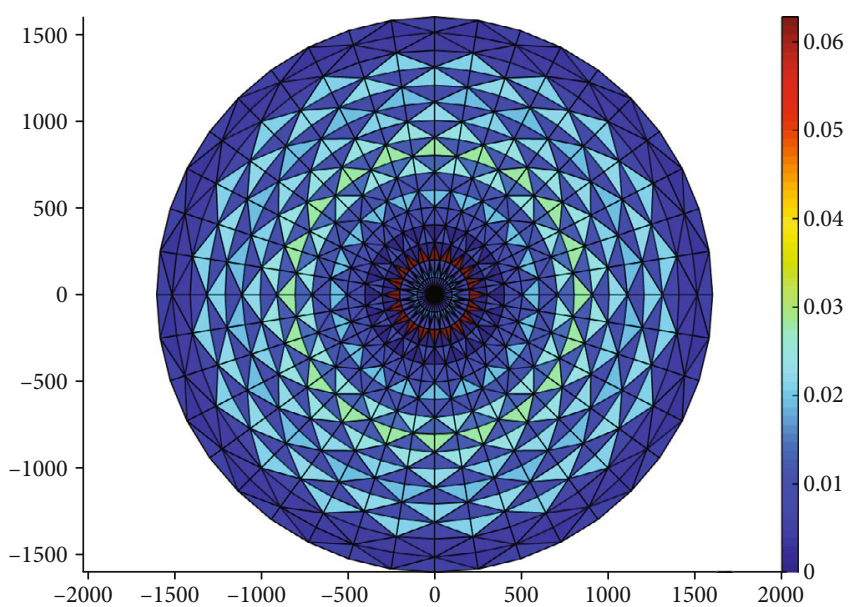

(a) Von Mises stress distribution

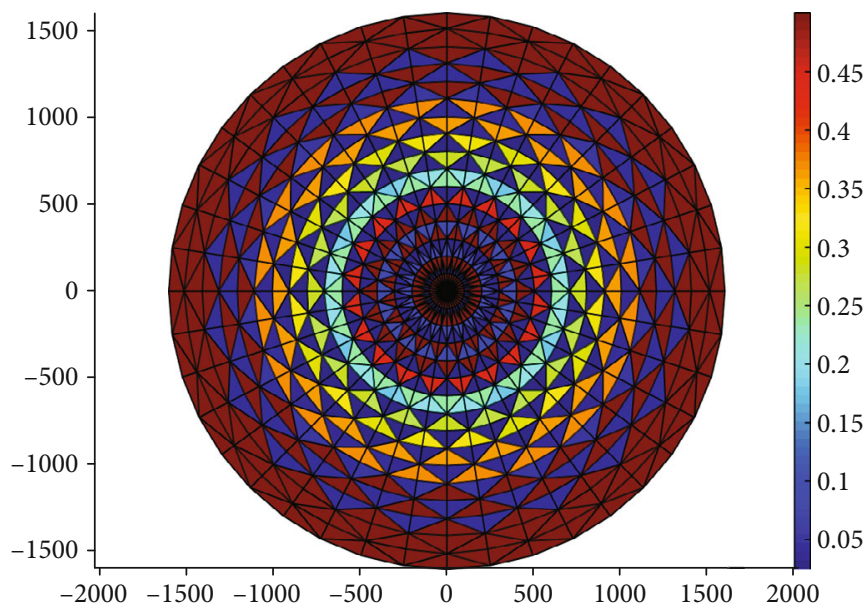

(b) Thickness distribution

FIGURE 5: Stress and thickness distribution of optimal reflector by the HPL-E Method.

TABLE 3: Result summary of the BFP-E Method by different parameter $r_{\min }$.

\begin{tabular}{lcc}
\hline$r_{\text {min }}(\mathrm{mm})$ & $\sigma_{\text {BFP-E }}(\mathrm{mm})$ & $\sigma_{\text {HPL-E }}(\mathrm{mm})$ \\
\hline 65.789 & 0.0326 & 0.4503 \\
98.684 & 0.0366 & 0.4492 \\
131.578 & 0.0435 & 0.4502 \\
197.368 & 0.0508 & 0.4515 \\
358.736 & 0.0544 & 0.4545 \\
\hline
\end{tabular}

Instead, the BFP-E Method can be relatively reasonable in the membrane sensitivity research. Even though the surface accuracy is not so sensitive to the reflector thickness in Ref. [10], the $\sigma_{\mathrm{BFP}-\mathrm{E}}$ based on the BFP-E evaluation method can be decreased substantially over the standard trial-and-error design approach, from $0.1489 \mathrm{~mm}$ to $0.0370 \mathrm{~mm}$ for the considered reflector profile. And the optimal objective of surface accuracy can be further improved with the development of element mesh refinement.

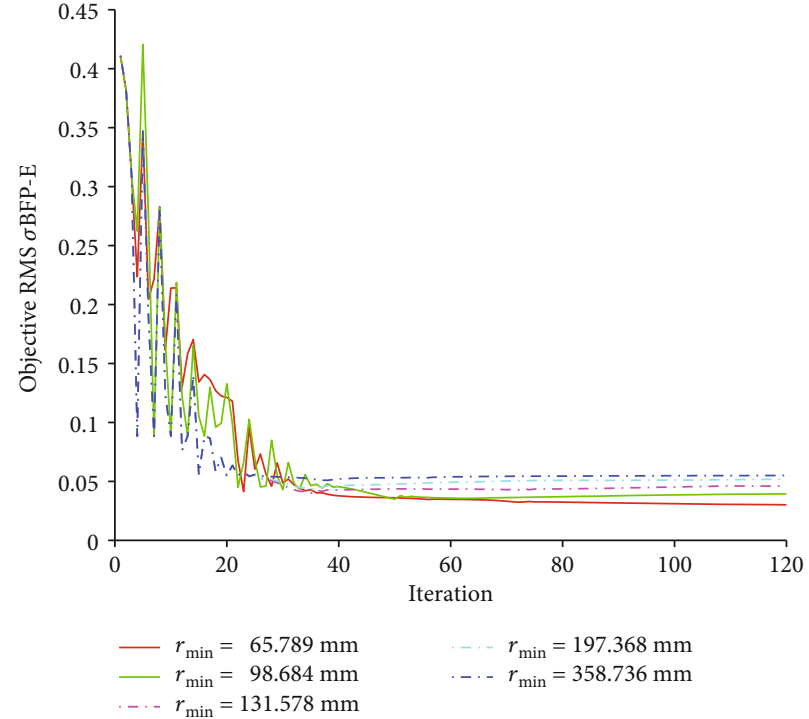

FIGURE 6: Iteration curves of reflector thickness optimization based on different filter radii. 


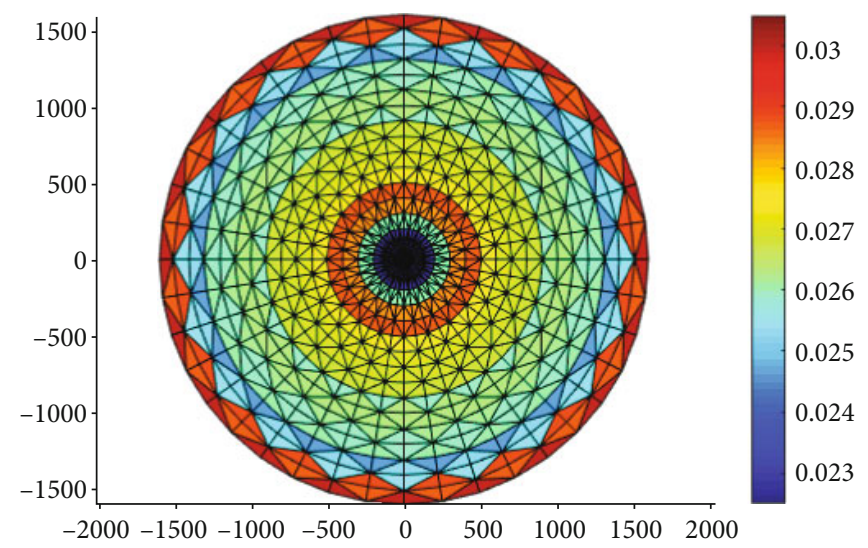

(a) $r_{\min }=65.789 \mathrm{~mm}$

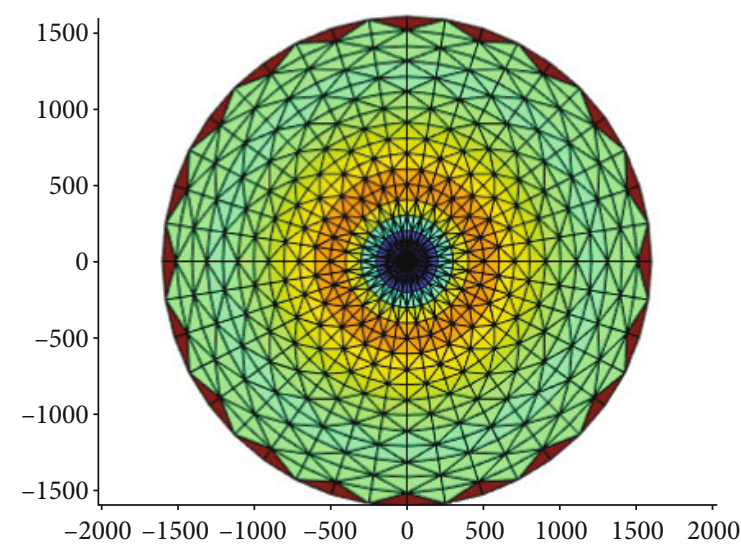

(b) $r_{\min }=98.684 \mathrm{~mm}$

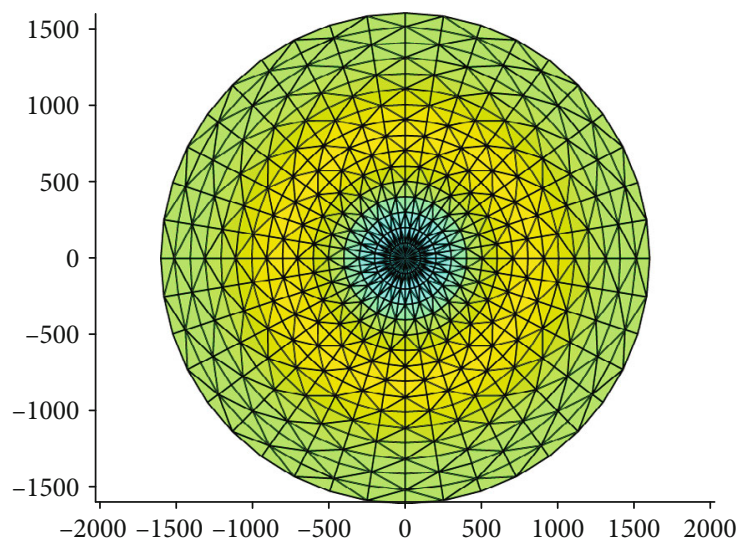

(d) $r_{\min }=197.368 \mathrm{~mm}$
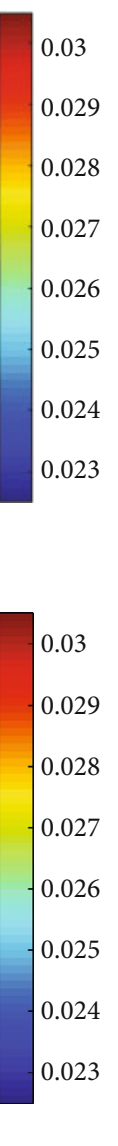

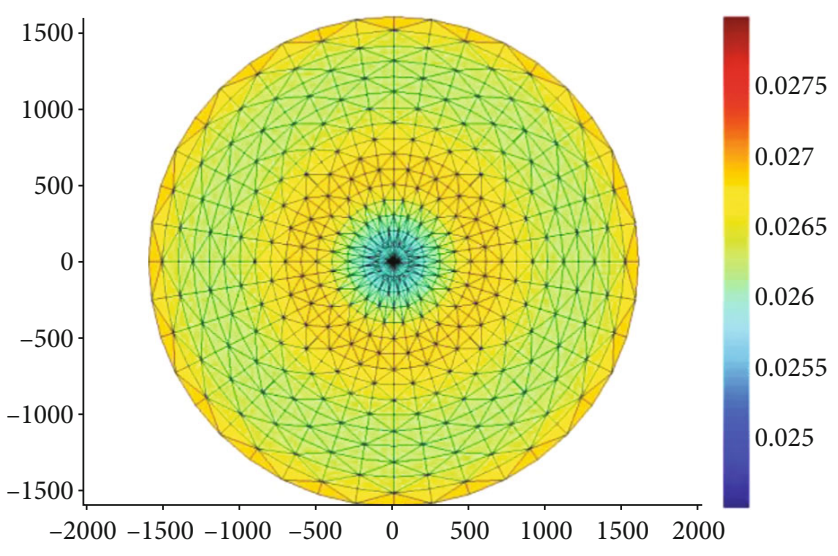

(c) $r_{\min }=131.578 \mathrm{~mm}$

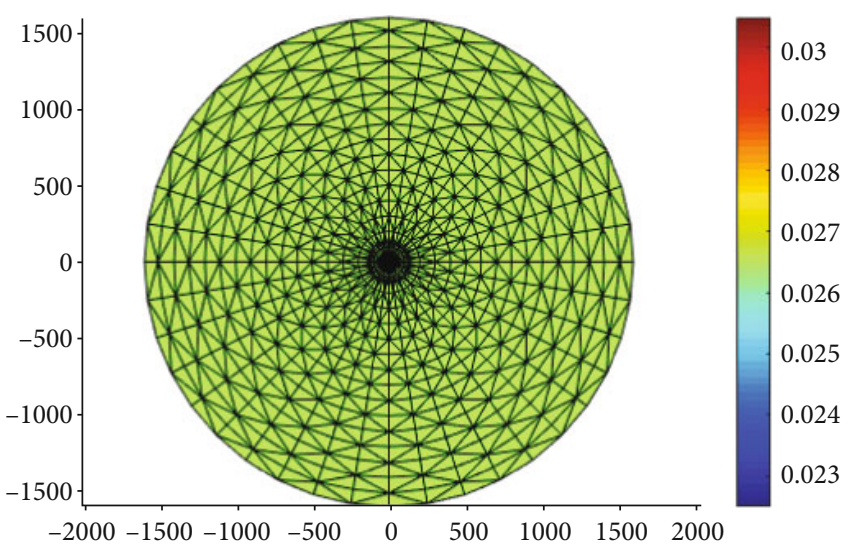

(e) $r_{\min }=358.736 \mathrm{~mm}$

FigurE 7: Effect of filter radius on the optimized reflector thickness distribution.

Thickness filtering controlled the degree of thickness variation, with larger filter radius giving solutions that may be more readily manufacturable at the expense of $\sigma_{\text {BFP-E }}$ performance. Even though there is little difference between the maximum and minimum thickness in the optimal thickness distribution, this small change can largely develop the surface accuracy of the membrane reflector.

Future work is aimed at improving the manufacturability of the reflector further based on the additional manufacturing technology, for example, using integer magnitudes of thickness through projection methods [29], and considering additional objectives, such as stiffness and dynamic factors.

\section{Data Availability}

Figure2.fig is used to support Figure 2 in the manuscript. Figure7(a).fig is used to support Figure 7 (a) $r_{\min }=65.789$ in the manuscript. Figure7(b).fig is used to support Figure 7(b) $r_{\min }=98.684$ in the manuscript. Figure7(c).fig is used to support Figure 7 (c) $r_{\min }=131.578$ in the manuscript. 
Figure7(d).fig is used to support Figure 7(d) $r_{\min }=197.368$ in the manuscript. Figure7(e).fig is used to support Figure $7(\mathrm{e}) r_{\min }=358.736$ in the manuscript. These data are the main results about this paper for support. However, further data like codes and programs cannot be released in public because this study is conducted for the project to figure out some actual problems. There is a confidentiality agreement for this part.

\section{Conflicts of Interest}

The authors declare that they have no conflicts of interest.

\section{Acknowledgments}

This research is supported by the China Scholarship Council (CSC), the National Science Foundation of China (Grant No. 11402229 and Grant No. 11902253), and the Zhejiang Provincial Natural Science Foundation of China (Grant No. LQ14A020003). The MMA optimizer is supplied by Professor Krister Svanberg. And Professor James K. Guest from Johns Hopkins University gave a great help in the derivation and program. The authors are grateful for these supports.

\section{References}

[1] G. G. Reibaldi and M. C. Bernasconi, "QUASAT program: the ESA reflector," Acta Astronautica, vol. 15, no. 3, pp. 181-187, 1987.

[2] R. E. Freeland, G. D. Bilyeu, and G. R. Veal, "Validation of a unique concept for a low-cost, lightweight space-deployable antenna structure," Acta Astronautica, vol. 35, no. 9-11, pp. 565-572, 1995.

[3] A. B. Chmielewski, M. Noca, and J. Ulvestad, "ARISE antenna," 2000, December 2011. http://trs-new.jpl.nasa.gov/ dspace/bitstream/2014/18857/1/99-2154.pdf.

[4] I. Eastwood, M. Thomson, H. Fang, J. Pearson, J. Moore, and J. Lin, "Prospects of large deployable reflector antennas for a new generation of geostationary Doppler weather radar satellites," in AIAA SPACE 2007 Conference \& Exposition, Long Beach, CA, USA, September 2007.

[5] H. Huang and F. Guan, "Mechanical study on initial profile solution for inflatable reflector made of orthotropic material," Acta Astronautica, vol. 104, no. 1, pp. 179-185, 2014.

[6] R. Levy, Structural engineering of microwave antennas for electrical, mechanical, and civil engineers, IEEE Press, Piscataway, NJ, USA, 1996.

[7] C. H. Jenkins and D. K. Marker, "Surface precision of inflatable membrane reflectors," Journal of Solar Energy Engineering, vol. 120, no. 4, pp. 298-305, 1998.

[8] G. Greschik, M. Mikulas, A. Palisoc, C. Cassapakis, and G. Veal, "Approximating paraboloids with axisymmetric pressurized membranes," in 39th AIAA/ASME/ASCE/AHS/ASC Structures, Structural Dynamics, and Materials Conference and Exhibit, Long Beach, CA, USA, April 1998.

[9] G. Greschik, M. M. Mikulas, and A. Palisoc, "Approximations and errors in pressurized axisymmetric membrane shape predictions," in 39th AIAA/ASME/ASCE/AHS/ASC Structures, Structural Dynamics, and Materials Conference and Exhibit, Long Beach, CA, USA, April 1998.
[10] G. Greschik, A. Palisoc, C. Cassapakis, G. Veal, and M. M. Mikulas, "Sensitivity study of precision pressurized membrane reflector deformations," AIAA Journal, vol. 39, no. 2, pp. 308314, 2001.

[11] G. Greschik, M. M. Mikulas, and A. Palisoc, "Torus-less inflated membrane reflector with an exact parabolic center," AIAA Journal, vol. 42, no. 12, pp. 2579-2584, 2004.

[12] S. Naboulsi, "Investigation of geometric imperfection in inflatable aerospace structures," Journal of Aerospace Engineering, vol. 17, no. 3, pp. 98-105, 2004.

[13] S. Kitano and R. Ishida, "An optimum thickness of inflatable reflector membrane," Journal of the Japan Society for Aeronautical and Space Sciences, vol. 56, no. 651, pp. 157-162, 2008.

[14] H. A. DeSmidt, K. W. Wang, and H. Fang, "Optimized gore/seam cable actuated shape control of gossamer membrane reflectors," Journal of Spacecraft and Rockets, vol. 44, no. 5, pp. 1122-1130, 2007.

[15] J. Hill, K. W. Wang, and H. Fang, "Advances of surface control methodologies for flexible space reflectors," Journal of Spacecraft and Rockets, vol. 50, no. 4, pp. 816-828, 2013.

[16] M. J. Coleman, F. Baginski, and R. R. Romanofsky, "Effect of boundary support and reflector dimensions on inflatable parabolic antenna performance," Journal of Spacecraft and Rockets, vol. 49, no. 5, pp. 905-914, 2012.

[17] R. Bouzidi and Y. Lecieux, "A numerical method to optimize the design of a space inflatable membrane reflector," Acta Astronautica, vol. 74, pp. 69-78, 2012.

[18] C. G. Wang, Z. M. Xia, and H. F. Tan, "Initial shape design and stability analysis of rib for inflatable deployable reflector," AIAA Journal, vol. 53, no. 2, pp. 486-492, 2015.

[19] Y. Luo, J. Xing, Y. Niu, M. Li, and Z. Kang, "Wrinkle-free design of thin membrane structures using stress-based topology optimization," Journal of the Mechanics and Physics of Solids, vol. 102, pp. 277-293, 2017.

[20] Y. Luo, Y. Niu, M. Li, and Z. Kang, "A multi-material topology optimization approach for wrinkle-free design of cablesuspended membrane structures," Computational Mechanics, vol. 59, no. 6, pp. 967-980, 2017.

[21] J. Xing, Y. Luo, J. Zhan, and Z. Kang, "Global shape optimization of fixtures to suppress wrinkles in large- displacement membrane structures," International Journal of Solids and Structures, vol. 144-145, pp. 301-312, 2018.

[22] K. Svanberg, "The method of moving asymptotes-a new method for structural optimization," International Journal for Numerical Methods in Engineering, vol. 24, no. 2, pp. 359-373, 1987.

[23] M. P. Bendsoe and O. Sigmund, Topology Optimization: Theory, Methods and Applications, Springer Science \& Business Media, 2003.

[24] J. Ruze, "Antenna tolerance theory-A review," Proceedings of the IEEE, vol. 54, no. 4, pp. 633-640, 1966.

[25] T. E. Bruns and D. A. Tortorelli, "Topology optimization of non-linear elastic structures and compliant mechanisms," Computer Methods in Applied Mechanics and Engineering, vol. 190, no. 26-27, pp. 3443-3459, 2001.

[26] A. Díaz and O. Sigmund, "Checkerboard patterns in layout optimization," Structural Optimization, vol. 10, no. 1, pp. 4045, 1995.

[27] B. Bourdin, "Filters in topology optimization," International Journal for Numerical Methods in Engineering, vol. 50, no. 9, pp. 2143-2158, 2001. 
[28] J. K. Guest, J. H. Prévost, and T. Belytschko, “Achieving minimum length scale in topology optimization using nodal design variables and projection functions," International Journal for Numerical Methods in Engineering, vol. 61, no. 2, pp. 238-254, 2004.

[29] A. T. Gaynor, N. A. Meisel, C. B. Williams, and J. K. Guest, "Multiple-material topology optimization of compliant mechanisms created via PolyJet three-dimensional printing," ASME Journal of Manufacturing Science and Engineering, vol. 136, pp. 1-10, 2014. 


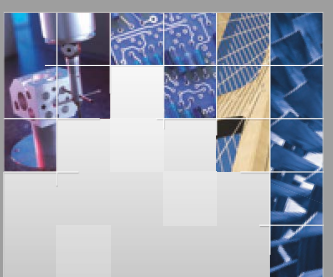

\section{Enfincering}
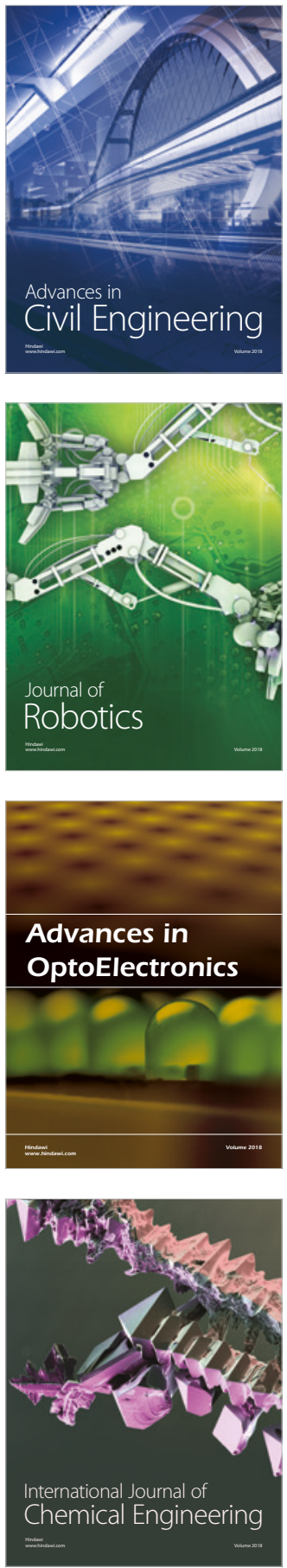

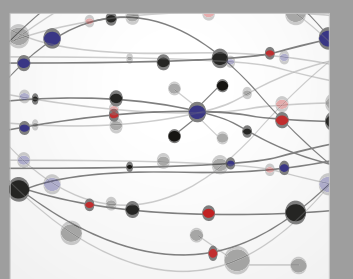

\section{Rotating \\ Machinery}

The Scientific World Journal

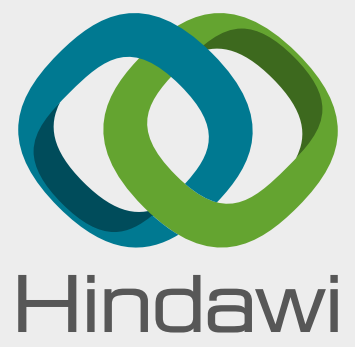

Submit your manuscripts at

www.hindawi.com
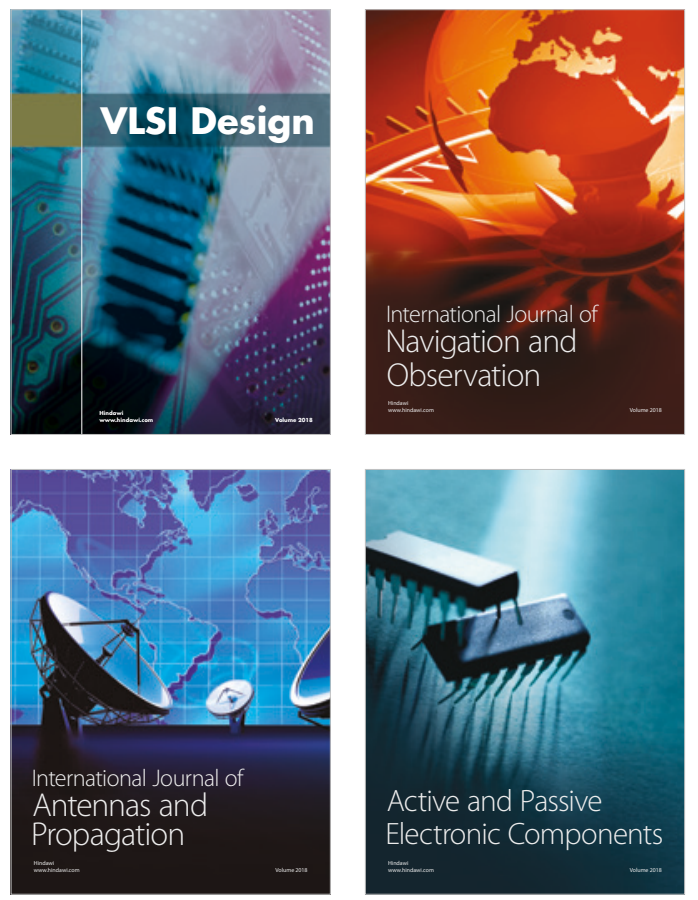
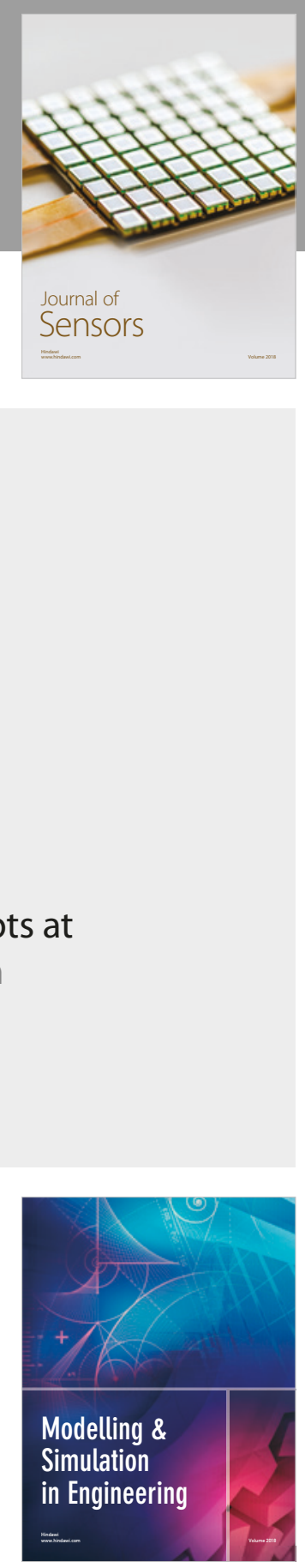

\section{Advances \\ Multimedia}
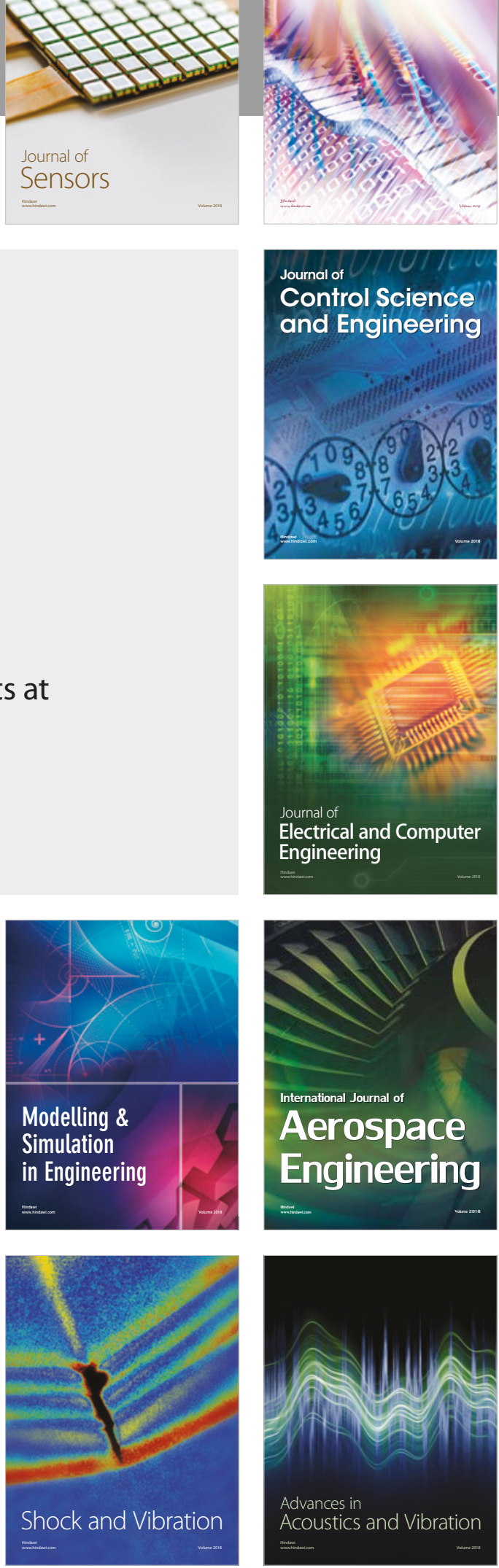